Claremont Colleges

Scholarship@ Claremont

All HMC Faculty Publications and Research

HMC Faculty Scholarship

3-1-1992

\title{
A Theoretical Investigation of the Ground and Low-Lying Excited States of Butadiene Radical Cation
}

\author{
Robert J. Cave \\ Harvey Mudd College \\ Michael G. Perrott '90 \\ Harvey Mudd College
}

\section{Recommended Citation}

A theoretical investigation of the ground and low-lying excited states of butadiene radical cation. Robert J. Cave and Michael G. Perrott, J. Chem. Phys. 96, 3745 (1992), DOI:10.1063/1.461879

This Article is brought to you for free and open access by the HMC Faculty Scholarship at Scholarship @ Claremont. It has been accepted for inclusion in All HMC Faculty Publications and Research by an authorized administrator of Scholarship @ Claremont. For more information, please contact scholarship@cuc.claremont.edu. 


\title{
A theoretical investigation of the ground and low-lying excited states of butadiene radical cation
}

\author{
Robert J. Cave ${ }^{\text {a) }}$ and Michael G. Perrott ${ }^{\text {b) }}$ \\ Department of Chemistry, Harvey Mudd College, Claremont, California 91711
}

(Received 7 August 1991; accepted 25 November 1991)

\begin{abstract}
Results are presented from $a b$ initio calculations on the ground and several low-lying excited states of the butadiene radical cation. In particular, we have calculated geometries for the ground and several excited states at the multiconfiguration self-consistent field level and characterized the planar stationary points. The vertical ionization potentials from the neutral molecule ground state and vertical excitation energies at the computed equilibrium geometry of the cation were also examined. A variety of methods were tested for the calculation of ionization potentials and excitation energies, including multiconfiguration self-consistent field, multireference singles and doubles configuration interaction (with and without size-

consistency correction), and multireference averaged coupled pair functional theory. It is found that several of the excited states are strong mixtures of so-called Koopmans' and nonKoopmans' configurations. In most cases, good agreement is found with experiment, but the excitation energy for the second excited $\pi$ state, a state which is multiconfigurational at zeroth order, is apparently overestimated at all levels of theory employed here.
\end{abstract}

\section{INTRODUCTION}

The field of polyene spectroscopy has been an active area for theory and experiment for the past 50 years and continues to produce intriguing results. Much of the work is motivated by the importance of polyenes in the areas of vision $^{1}$ and of conducting polymers, ${ }^{2}$ but current work is also concerned with the unusual properties of the shorter chain polyenes. ${ }^{3}$

The interest in the neutral polyenes arises largely from the finding that fluorescence occurs not from the lowest optically allowed state (which is of $\pi \rightarrow \pi^{*}$ character), but from a nominally doubly excited, one-photon-forbidden state. ${ }^{3,4}$ The fluorescence intensity from the doubly excited state increases with decreasing chain length down to octatetraene (at least in solution $)^{5}$ and then vanishes for hexatriene and butadiene. ${ }^{6}$ Although fluorescence from the doubly excited state is not observed for butadiene, a variety of experiments have estimated the position of this state using multiphoton ionization ${ }^{7.8}$ or electron scattering. ${ }^{9,10}$ Experimental studies of hexatriene ${ }^{7}$ and octatetraene ${ }^{11}$ appear to indicate that the minimum of the doubly excited state is similar to that of the ground state.

Theoretical studies of the straight chain polyenes have also been attempted. Semiempirical methods obtain good agreement with experiment for the position of the first optically allowed state and predict the doubly excited state to fall below the $\pi \rightarrow \pi^{*}$ state in all cases. ${ }^{12} A b$ initio results have predicted the $\pi \rightarrow \pi^{*}$ state to lie above that observed experimentally and to be a valence-Rydberg mixture at the ground state geometry. ${ }^{13-18}$ Also, $a b$ initio results have predicted that the doubly excited state lies above the $\pi \rightarrow \pi^{*}$ state for hexatriene ${ }^{17(b)}$ and butadiene. ${ }^{13-18}$ Finally, both semiempir-

" Author to whom correspondence should be addressed.

b) Current address: Department of Chemistry, University of California at Berkeley, Berkeley, CA 94720. ical and ab initio methods that have examined geometry relaxation effects in the shorter polyenes indicate that the doubly excited state is very sensitive to changes in geometry and that its energy decreases strongly as the bond alternation is reversed. ${ }^{12(c), 18,19}$ For octatetraene, less theoretical work has been done, but the work that is currently available predicts that the energy decrease upon rearrangement is as large as that found in the shorter chain polyenes. ${ }^{12(c), 20}$

The butadiene cation has also been studied extensively experimentally ${ }^{21-27}$ and theoretically. ${ }^{28-34}$ Experimentally, the molecule is of intrinsic interest as a model for systems where ionization occurs to states of mixed character (mixing of Koopmans' and non-Koopmans' states) ${ }^{25}$ In addition, it is a component of prototype addition reactions involving conjugated ions. ${ }^{27}$ Theory has been used to interpret the ionization or UV spectra and the nature of the mixing between zeroth-order configurations in the excited states has been discussed. ${ }^{25,28-34}$ Much of past theory has been semiempirical in nature $e^{25-32}$ and has tended to be more accurate than the available $a b$ initio work. ${ }^{33,34}$ In any event, several questions remain unanswered concerning the ground and excited states of the radical cation, including the detailed geometry of the ground state, the nature of other stationary points for the ground state, the geometries of the low-lying excited states, details regarding the compositions of the excited states, and why past $a b$ initio work has been inaccurate in the calculation of excitation energies. In addition, one might ask whether the intensity maxima correspond to vertical transitions, or whether significant spectral intensity arises from nonvertical transitions as has been suggested for the neutral species. ${ }^{17(a)}$ In this paper, we address some of these questions using ab initio methods.

We have also used the butadiene radical cation as a model for comparing various levels of theory. One aim is to seek what might be considered a "minimal" level of theory necessary for treating the ground and low-lying excited states of 
the cation. This will be of use in examining larger polyene radical cations, as well as larger regions of the potential energy surface for the butadiene cation. We also compare excitation energies obtained using multireference singles and doubles configuration interaction (MRSDCI) (with and without correction for size inconsistency) and the multireference averaged coupled pair functional (MRACPF) theo$\mathrm{ry}^{35}$ of Gdanitz and Ahlrichs. Single-reference and multireference configuration interaction methods have been successful in describing excited states in small molecules; ${ }^{36}$ here we examine the accuracy of $\mathrm{CI}$ for the calculation of excitation energies where size-inconsistency ${ }^{37}$ effects are expected to be significant. Averaged coupled-pair functional theory is a nearly size-consistent modification to the MRSDCI equations, but is nonvariational. Thus these calculations assess its utility for the calculation of excitation energies in large molecules where significant zeroth-order mixing occurs in the description of the excited states. It is this zeroth-order mixing which makes the excited states a less tractable problem for single reference methods.

The outline of the remainder of the paper is as follows: In Sec. II, the theoretical methods and geometries used are outlined. In Sec. III, we present our results and in Sec. IV we compare the results with experiment and past theory. We also compare the present methods with each other.

\section{THEORETICAL METHODS}

All geometry optimizations yielded $C_{2 h}$ geometries for the ground state of the cation; experiment has shown that the neutral molecule is also $C_{2 h} \cdot{ }^{38}$ To simplify the discussion, we will refer to orbitals and states that are symmetric with respect to reflection in the molecular plane as " $\sigma$ " $\left(a_{g}\right.$ and $b_{u}$ in $C_{2 h}$ ) and orbitals or states that are antisymmetric with respect to reflection in the molecular plane as " $\pi$ " $\left(a_{u}\right.$ and $b_{g}$ in $C_{2 h}$ ).

Several basis sets were used in the present study. For geometry optimizations on the ground and low-lying $\pi$ states, the Slater-type orbital STO-3G basis ${ }^{39}$ was used, augmented in some cases with a set of $p$ diffuse functions on each carbon atom $(\alpha=0.034$, denoted STO-3 $+\mathrm{G})$. Geometry optimizations were also performed on the ground state in the 6-31G basis. ${ }^{40}$ In some cases, for the purpose of calculating excitation energies, we have also augmented the 6-31G basis with $p$ diffuse functions on carbon (denoted $6-31+\mathrm{G}$ ) or polarization functions on carbon $(\alpha=0.8),{ }^{40(c)}$ denoted 6 31G*. For the MRSDCI and MRACPF calculations, the basis sets used for $\mathbf{C}$ and $\mathbf{H}$ were the Dunning/Huzinaga double zeta basis sets, ${ }^{41}$ augmented with polarization functions (carbon $d, \alpha=0.75$; hydrogen $p, \alpha=1.00$ ) $^{41(\mathrm{c})}$ and $\pi$ diffuse functions on carbon $(\alpha=0.034) .^{41(c)}$ This basis is denoted below as DZP + . For one series of test calculations, we removed the polarization functions from the DZP + basis; this smaller basis is denoted $\mathrm{DZ}+$.

To estimate vertical ionization potentials, we have performed calculations at the experimental ground state geometry for the neutral species of Haugen and Traetteberg. ${ }^{38} \mathrm{We}$ are unaware of any detailed structural data for the ion [however, recent electron-nuclear double resonance (ENDOR) work $^{26}$ indicates that the ground state is s-trans ], so we per- formed geometry optimizations for the ground and excited states at the multiconfiguration self-consistent field (MCSCF) level. It was found that single-reference SCF calculations on the ion's ground state gave rise to a symmetry breaking that disappeared when the $\pi$ electrons were correlated; thus we chose a minimal level of treatment for all $\pi$ hole states as all configurations having the three $\pi$ electrons in four $\pi$ orbitals (two $a_{u}$, two $b_{g}$ in $C_{2 h}$ symmetry). These complete active space SCF (CASSCF) calculations are denoted below as 3/4 CASSCF to indicate that three electrons are correlated in four orbitals. In the case of $\sigma$-hole states, we have performed CASSCF calculations with a singly occupied $\sigma$ orbital of the proper symmetry and allowed all four $\pi$ electrons to be distributed over the four lowest $\pi$ orbitals; these are denoted below as 4/4 CASSCF results. There is an inherent bias here towards lowering the $\sigma$ states relative to the $\pi$ states since four electrons are correlated in these ion states, rather than three in the $\pi$ states. It will be seen in comparison with more extended correlation treatments below that the effect is not large.

In the calculations of vertical excitation energies for the ion, we have used the 6-31G 3/4 CASSCF optimized ground state $\left(1^{2} B_{g}\right)$ geometry. At the CASSCF level, we have also examined nonvertical excitation energies using the geometries obtained for the excited states in the STO-3 + G geometry optimizations.

Calculations of the ionization potentials and excitation energies for the ion were also performed using MRSDCI and MRACPF. The point of departure for both methods is the choice of a set of configurations that are deemed to describe the state of interest at zeroth order. The same reference space was used for both methods. The reference space was generally built up by performing MRACPF calculations with small reference spaces and adding important nonreference configurations from the ACPF wave function. This process was iterated (normally twice) until it was deemed that no further expansion of the reference space would affect the state energy significantly.

Once the reference space was chosen, all single and double excitations (except from the doubly occupied carbon $1 \mathrm{~s}$ orbitals) from all configurations in the reference space were scanned using second-order Rayleigh-Schrödinger perturbation theory. Those passing a specified threshold for energy lowering were included in the variational portion of the calculation. Except where noted, for calculations on the lowest state of a given symmetry, we performed perturbation theory selection using only the lowest root of the zeroth-order Hamiltonian. For the second state in a given symmetry, perturbation theory selection was performed using the two lowest roots of the zeroth-order Hamiltonian. The effects of the configurations not treated variationally are included using the extrapolation procedure given in Eq. (1), which has been presented previously ${ }^{42}$

$E_{\text {method }}^{\text {ex }}=E_{0}+\left(E_{\text {method }}-E_{0}\right)(1+\mathrm{PTD} / \mathrm{PTK})$.

In Eq. (1), $E_{\text {method }}^{\text {ex }}$ is the extrapolated singles and doubles energy and $E_{\text {method }}$ is the energy obtained from the calculation on the subset of all single and double excitations. Pertur- 
bation theory energy kept (PTK) is the energy lowering at second order for the configurations treated variationally, perturbation theory energy discarded (PTD) is the analogous quantity for those configurations not included in the variational treatment. $E_{0}$ is the zeroth-order energy obtained from diagonalizing the Hamiltonian over the set of reference configurations, choosing the root corresponding to the state of interest.

We have also calculated approximately size-consistent results using a correction due to Davidson and Silver. ${ }^{43,44}$ This correction takes approximate account of the effects of quadruple excitations in the CI wave function (hence the designation $+Q$ ) and of the deviation of the coefficient of the zeroth-order wave function from unity in the final CI wave function. The expression used here is

$$
E_{\mathrm{SDCl}+\mathrm{Q}}^{\mathrm{ex}}=E_{0}+\frac{\Sigma_{i} c_{0 i}^{2}}{2 \Sigma_{i} c_{0 i}^{2}-1}\left(E_{\mathrm{SDCl}}^{\mathrm{ex}}-E_{0}\right),
$$

where $E_{\mathrm{SDCI}+\mathrm{Q}}^{\mathrm{ex}}$ is the extrapolated singles and doubles CI energy corrected for the approximate contribution of quadruple excitations, $E_{\mathrm{SDCl}}^{\text {ex }}$ is defined above, and $\Sigma_{i} c_{0 i}^{2}$ is the sum of the squares of all reference-space-configuration coefficients in the final wave function.

The ACPF equations have been developed and discussed previously by Gdanitz and Ahlrichs. ${ }^{35}$ For the present purposes, it is important to note that ACPF attempts to include the effects of quadruple and higher configurations by modifying the SDCI energy functional. As a result, near size consistency is achieved, but at the cost of yielding a nonvariational procedure. Nevertheless, ACPF has been shown to produce quite accurate total energies and excitation energies in previous studies. ${ }^{35,45}$ The method can be applied as a single-reference or multireference approach and it is the multireference nature of the method which is necessary for many of the states considered here. In a previous study, it has been shown that the perturbation theory extrapolation procedure of Eq. (1) yields quite accurate results for $\mathrm{ACPF}^{45}$ and quasidegenerate variational theory. ${ }^{45,46}$

In the MRSDCI and MRACPF calculations, we used restricted open-shell Hartree-Fock (ROHF) orbitals as the starting point for the calculation. The virtual orbitals used were the $K$ orbitals $(K O)$ of Feller and Davidson. ${ }^{47}$ In the case of the ground state of the ion $\left(1^{2} B_{g}\right.$ in $\left.C_{2 h}\right)$ and the two low-lying $\sigma$-hole states $\left(1^{2} B_{u}\right.$ and $\left.1^{2} A_{g}\right)$, these orbitals were used for the remainder of the calculation. At the ROHF level, the highest occupied molecular orbital (HOMO) is of $b_{g}$ symmetry, having a planar node and an additional node perpendicular to the molecular plane between the two central carbons. The HOMO-1 is of $a_{u}$ symmetry, having only a planar node. In the case of excited $\pi$ states, we used natural orbitals ${ }^{48}$ to obtain better zerothorder approximations for the orbitals. For the $2^{2} B_{8}$ state, a $\pi$-full CI was performed beginning with the $1^{2} B_{g}$ ROHF/ KOs and the natural orbitals (NO) were formed for the second root of the CI matrix. Using these orbitals, a second CI was performed, including all $\sigma$ single excitations from all configurations having the three $\pi$ electrons distributed over the four lowest $\pi$ orbitals. The natural orbitals for the second root of this matrix were obtained and all further $2{ }^{2} B_{g}$ calculations were based on these NOs. For the ${ }^{2} A_{u}$ states, an entirely analogous procedure was used, except that the average natural orbitals ${ }^{49}$ (ANO) were formed from the two lowest roots of each CI matrix. This has proven to be an effective procedure in calculations on the neutral polyenes for the balanced treatment of several low-lying states of the same symmetry. ${ }^{17,19,20}$

Geometry optimizations and MCSCF results were obtained using GAMESS. ${ }^{50}$ All MRSDCI and MRACPF calculations were performed using MELDF. ${ }^{51}$

\section{RESULTS}

In Table I, results are presented from CASSCF geometry optimizations in the STO-3G and 6-31G basis sets for the trans and cis geometries of the cation ground state $\left(1^{2} B_{g}\right)$. Figure 1 presents the numbering scheme used to report the molecular geometries. For comparison, we also present the experimental geometry for the neutral molecule. ${ }^{38}$ As one might expect, based on ionization from a $b_{g} \pi$ orbital with the character discussed above, the central bond shortens and the two terminal bonds lengthen relative to the neutral molecule. Results from analogous CASSCF calculations on the neutral species in the STO-3G basis reproduce the bond al-

TABLE I. $1^{2} B_{2}$ geometries for $\mathrm{C}_{4} \mathrm{H}_{6}^{+}$.

\begin{tabular}{llllll}
\hline \hline Length/angle" & STO-3G trans & $6-31 \mathrm{G}$ trans & STO-3G cis & $6-31 \mathrm{G}$ cis & Neutral $^{\mathrm{b}}$ \\
\hline $\mathrm{C}_{1}-\mathrm{C}_{2}$ & 1.404 & 1.390 & 1.403 & 1.390 & 1.343 \\
$\mathrm{C}_{2}-\mathrm{C}_{2^{\prime}}$ & 1.419 & 1.400 & 1.425 & 1.407 & 1.467 \\
$\mathrm{H}_{1}-\mathrm{C}_{1}$ & 1.092 & 1.073 & 1.089 & 1.071 & 1.094 \\
$\mathrm{H}_{2}-\mathrm{C}_{1}$ & 1.091 & 1.072 & 1.091 & 1.071 & 1.094 \\
$\mathrm{H}_{3}-\mathrm{C}_{2}$ & 1.092 & 1.075 & 1.092 & 1.074 & 1.094 \\
$\Theta_{\mathrm{C}_{1} \mathrm{C}_{2} \mathrm{C}_{2^{\prime}}}$ & 121.4 & 122.1 & 124.9 & 125.4 & 122.8 \\
$\Theta_{\mathrm{H}_{1} \mathrm{C}_{1} \mathrm{C}_{2}}$ & 121.0 & 121.5 & 117.1 & 123.1 & 119.5 \\
$\Theta_{\mathrm{H}_{2} \mathrm{C}_{1} \mathrm{C}_{3}}$ & 120.9 & 121.2 & 122.8 & 120.3 & 119.5 \\
$\Theta_{\mathrm{H}_{1} \mathrm{C}_{2} \mathrm{C}_{2^{\prime}}}$ & 119.1 & 119.0 & 119.8 & 116.9 & 117.7 \\
\hline
\end{tabular}

\footnotetext{
Bond lengths in Angstroms, bond angles in degrees. The 3/4 CASSCF energies are trans-STO-3G, -152.8352 hartrees; $6-31 \mathrm{G},-154.6179$ hartrees; cis-STO-3G, -152.8310 hartrees, $6-31 \mathrm{G},-154.6125$ hartrees.

${ }^{b}$ Reference 38.
} 


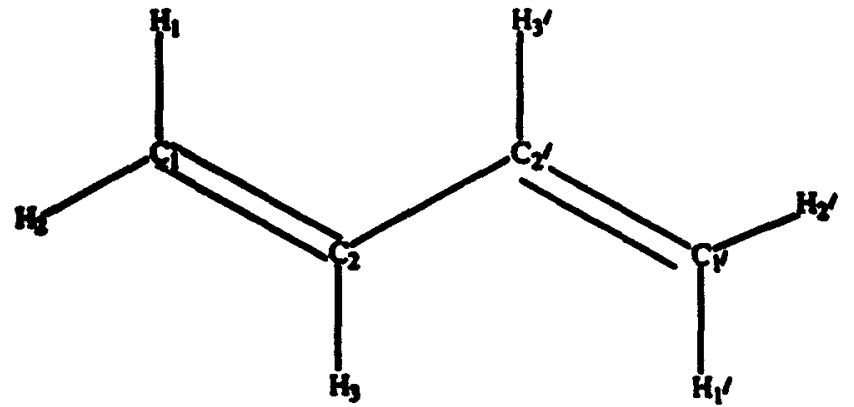

s-trans 1,3 Butadiene

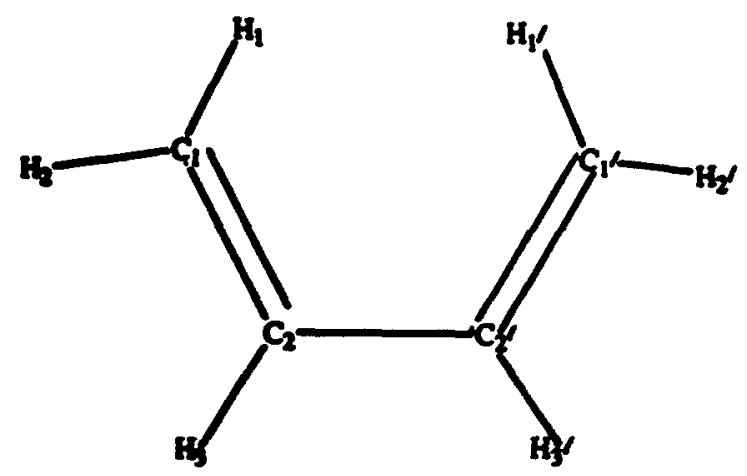

s-cis 1,3 Butadiene

FIG. 1. The numbering scheme used for geometry data in Tables I and IV.

ternation seen experimentally; ${ }^{52}$ thus we believe the present results are not artifactual. While some differences are observed, overall the two basis sets yield similar results. The bond angles are all near $120^{\circ}$ as expected. The $s$-trans structure is predicted to be below the $s$-cis structure by from 2.5 to $3.5 \mathrm{kcal} / \mathrm{mol}$. Geometry optimizations were attempted beginning from torsional angles about the central $\mathrm{C}-\mathrm{C}$ bond not equal to $0^{\circ}$ or $180^{\circ}$; in these cases, the optimized geometry was found to be either s-trans or s-cis, suggesting that no gauche minima exist for the cation. In the neutral molecule, the relative energetics of s-cis and gauche structures are highly sensitive to basis set and correlation treatment. ${ }^{53}$ This is less likely to be the case for the cation since the ionization to create the $1^{2} B_{g}$ state removes an electron that is antibonding across the central $\mathrm{C}-\mathrm{C}$ bond. Thus one expects the torsional potential to rise significantly as one twists from planar in either the trans or the cis geometry.

Vertical ionization potentials and excitation energies to several low-lying $\pi$ states are presented at the CASSCF level in Table II. The ionization potentials were calculated at the experimental geometry for the neutral molecule and the vertical excitation energies were calculated at the 6-31G CASSCF geometry for the ground state of the ion. No significant changes would occur had the STO-3G CASSCF geometry been used instead. Vertical excitation energies were examined in four different basis sets. All excitation energies are
TABLE II. Ionization potentials and excitation energies from $\pi$ CASSCF calculations.

\begin{tabular}{|c|c|c|c|c|}
\hline State & STO $-3+\mathrm{G}$ & $6-31 G$ & $6.31+G$ & $6-31 G^{*}$ \\
\hline \multicolumn{5}{|c|}{ Ionization potentials $\mathrm{s}^{\mathrm{a}}$} \\
\hline $1^{2} B_{8}$ & & $8.55(-)$ & & \\
\hline $1^{2} A_{u}$ & & $11.02(2.47)$ & & \\
\hline $2^{2} A_{\mu}$ & & $13.72(5.17)$ & & \\
\hline $2^{2} B_{8}$ & & $15.45(6.90)$ & & \\
\hline $1^{2} A_{g}$ & & $12.22(3.67)$ & & \\
\hline $1^{2} B_{k}^{\circ}$ & & $14.11(5.56)$ & & \\
\hline \multicolumn{5}{|c|}{ Excitation energies ${ }^{b}$} \\
\hline $1^{2} A_{k}$ & 2.73 & 2.51 & 2.51 & 2.48 \\
\hline $2^{2} A_{u}$ & 5.22 & 4.96 & 4.92 & 5.07 \\
\hline $2^{2} B_{g}$ & 7.29 & 6.80 & 6.67 & 6.89 \\
\hline $1^{2} A_{g}$ & & 4.58 & 4.77 & \\
\hline $1^{2} B_{u}$ & & 6.10 & 6.30 & \\
\hline
\end{tabular}

"Ionization potentials are in electron-volts relative to a 4/4 CASSCF calculation for the $1^{\prime} A_{g}$ state in the given basis. The experimental equilibrium geometry for the $1^{\prime} A_{\mathrm{g}}$ state (Ref. 38) was used for all states. The total energy for the $1^{1} A_{g}$ state is $6-31 \mathrm{G},-154.9214$ hartrees. For ease of comparison, excitation energies for ion states relative to the $1^{2} B_{g}$ state at this geometry are also included in parentheses.

${ }^{6}$ Excitation energies are in electron-volts relative to the $3 / 4$ CASSCF calculation for the $1{ }^{2} B_{g}$ state in the given basis. In all cases, the $6-31 \mathrm{G} 3 / 4$ CASSCF ground state geometry was used. The total energies for the $1{ }^{2} B_{g}$ state are STO-3 + G, -152.8603 hartree; $6-31 \mathrm{G},-154.6179$ hartree; 6 $31+\mathrm{G},-154.6190$ hartree; 6-31G*, -154.6746 hartree.

lowered in proceeding from STO-3 + G to the 6-31G basis; no large change is brought about by inclusion of diffuse functions in the 6-31G basis. One might have expected this, since for the cation the Rydberg states will be much higher in energy than the low-lying valence states due to the high I.P. of the cation. Addition of polarization functions at the CASSCF level also has limited effect on the excitation energies. It will be seen below that the larger basis set results are in better agreement with extended calculations, as well as with experiment, but it should be noted that the STO-3G results yield the correct ordering, state characters, and approximate energy separations.

Comparing the ion state energy differences at the neutral ground state geometry and ion ground state geometry, one sees that the $\pi$ excitation energies exhibit small changes with geometry, but the $\sigma$ excitation energies change to a much larger extent. This is due largely to the character of the orbitals out of which the electron is taken for the $\sigma$ states. For the $1{ }^{2} B_{u}$ state, the electron is removed from an orbital that is bonding between the carbons and hydrogens, but has no appreciable density between the carbons. In this case, one would expect similar C-C bond lengths as are found in the neutral and the bond length changes in proceeding to the cation geometry should lead to a significant increase in energy. In the case of the $1^{2} A_{g}$ state, which has significant $\mathrm{C}-\mathrm{H}$ bonding and also $\mathrm{C}-\mathrm{C}$ bonding character between the central carbons, similar reasoning applies. One might expect a larger effect here, since the cation geometry has a shortened central $\mathrm{C}-\mathrm{C}$ bond relative to the neutral, but the electron 
TABLE III. Natural orbital occupations and wave function expansions for the ground and excited states of $\mathrm{C}_{4} \mathrm{H}_{6}^{+}$from CASSCF wave functions."

\begin{tabular}{|c|c|c|c|c|c|c|}
\hline \multicolumn{7}{|c|}{ Natural orbital occupations } \\
\hline State & $6 b_{*}$ & $7 a_{8}$ & $1 a_{\mu}$ & $1 b_{g}$ & $2 a_{\mu}$ & $2 b_{g}$ \\
\hline $1^{2} B_{2}$ & 2.00 & 2.00 & 1.92 & 0.99 & 0.066 & 0.022 \\
\hline $1^{2} A_{*}$ & 2.00 & 2.00 & 1.22 & 1.38 & 0.38 & 0.017 \\
\hline $2^{2} A_{k}$ & 2.00 & 2.00 & 1.52 & 0.75 & 0.68 & 0.049 \\
\hline $2^{2} B_{8}$ & 2.00 & 2.00 & 1.59 & 0.30 & 0.30 & 0.82 \\
\hline $1^{2} A_{s}$ & 2.00 & 1.00 & 1.92 & 1.83 & 0.17 & 0.08 \\
\hline $1^{2} B_{k}$ & 1.00 & 2.00 & 1.92 & 1.83 & 0.18 & 0.08 \\
\hline \multicolumn{7}{|c|}{ 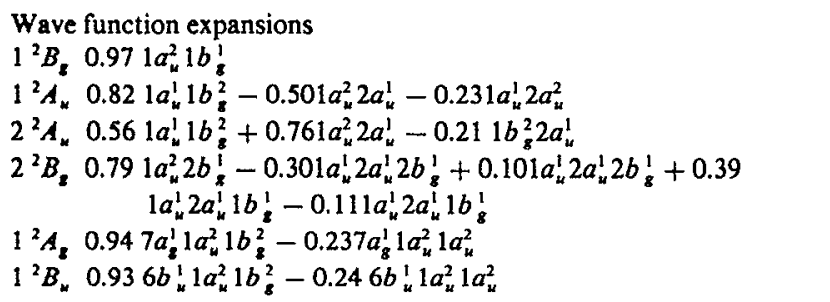 } \\
\hline
\end{tabular}

- Results from $3 / 4$ or $4 / 4$ CASSCFs in the 6-31G basis. The calculated ion ground state geometry is used in all cases. The wave function expansion is performed in the natural orbitals for each state and only configuration coefficients larger than 0.2 are included above, except for case of multiple spin couplings. Unless indicated, there are 13 doubly occupied $\sigma$ orbitals $\left(1-7 a_{z}\right.$ and $\left.1-6 b_{w}\right)$. See the text for further details.

removed would lead to a lengthening of this bond. It will be shown below that the more extensive correlation treatments reproduce this behavior for the $\pi$ and $\sigma$ states.

It has been noted previously ${ }^{25}$ that the $\pi$ excited states of the cation are multiconfigurational, even at zeroth order. Support for this assertion has often been taken from the large contribution of several configurations in semiempirical or $a b$ initio CI wave functions. ${ }^{30,32,34}$ While this can be an indicator of multiconfigurational character, it is not determinative in and of itself, since the orbitals used in these expansions are generally not optimized for the state of interest, but instead are the occupied and virtual orbitals for the ground state. If canonical Hartree-Fock virtual orbitals are used, they will be too diffuse to describe excited state valence orbitals and the expansion of the state will appear to have several large terms. A better method of assessing the presence or absence of multiconfigurational character in a state is to form the natural orbitals for the state and examine the wave function expansion in the natural orbitals, as well as the NO occupations. ${ }^{48}$ The natural orbitals yield the most compact full CI description of a state and tend to yield compact descriptions of truncated CIs. ${ }^{48}$ In Table III, NO occupations and wave function expansions are presented for the $\sigma$ and $\pi$ states considered here. The NOs in each case are formed from the wave function expansion in the optimum orbitals for the given state. For states that are basically single configurational at the zeroth-order level one sees orbital occupations near one or two. On the other hand, for multiconfigurational states, one sees several occupations that are clearly nonzero in the $\pi$ space. From the wave function expansions for the $1^{2} A_{u}$ and $2^{2} A_{u}$ states, one can see both Koopmans' configurations $\left(1 a_{u}^{1} 1 b_{g}^{2}\right)$ and non-Koopmans' configurations $\left(1 a_{u}^{2} 2 a_{u}^{1}\right)$ entering with significant weights.
TABLE IV. Ground and excited state geometries for $\mathrm{C}_{4} \mathrm{H}_{6}^{+}$: $^{*}$

\begin{tabular}{lcccc}
\hline \hline Length/angle $^{\mathrm{b}}$ & $1^{2} B_{\mathrm{g}}$ & $1^{2} A_{\mathrm{u}}$ & $2^{2} A_{u}$ & $2^{2} B_{g}$ \\
\hline $\mathrm{C}_{1}-\mathrm{C}_{2}$ & 1.412 & 1.446 & 1.480 & 1.495 \\
$\mathrm{C}_{2}-\mathrm{C}_{2^{\prime}}$ & 1.426 & 1.472 & 1.465 & 1.535 \\
$\mathrm{H}_{1}-\mathrm{C}_{1}$ & 1.092 & 1.088 & 1.096 & 1.095 \\
$\mathrm{H}_{2}-\mathrm{C}_{1}$ & 1.092 & 1.088 & 1.096 & 1.097 \\
$\mathrm{H}_{3}-\mathrm{C}_{2}$ & 1.091 & 1.095 & 1.087 & 1.086 \\
$\Theta_{\mathrm{C}_{1} \mathrm{C}_{2} \mathrm{C}_{2^{\prime}}}$ & 121.6 & 123.6 & 121.6 & 116.7 \\
$\Theta_{\mathrm{H}_{1} \mathrm{C}_{1} \mathrm{C}_{2}}$ & 121.3 & 121.2 & 121.5 & 120.8 \\
$\Theta_{\mathrm{H}_{2} \mathrm{C}_{1} \mathrm{C}_{2}}$ & 120.7 & 119.6 & 120.4 & 121.0 \\
$\Theta_{\mathrm{H}_{3} \mathrm{C}_{2} \mathrm{C}_{2}}$ & 119.1 & 117.5 & 120.3 & 122.0 \\
\hline \hline
\end{tabular}

a Results from 3/4 CASSCF in the STO-3 + G basis. The molecule is assumed to have $C_{2 h}$ symmetry.

${ }^{b}$ Bond lengths are in Angstroms, bond angles in degrees. The 3/4 CASSCF energies at the optimum geometry for the given state are $1^{2} B_{g}-152.8637$ hartrees; $1{ }^{2} A_{\alpha}-152.7735$ hartrees; $2{ }^{2} A_{\alpha}-152.6898$ hartrees; $2{ }^{2} B_{s}$ -152.6321 .

In Table IV, optimized planar geometries are presented for the $\pi$ excited states considered above. The geometries were obtained at the 3/4 CASSCF level in the STO-3 + G basis. For these results, we reoptimized the ground state of the ion in the STO- $3+\mathrm{G}$ basis. The energies of the $2{ }^{2} B_{8}$ and $2{ }^{2} A_{u}$ states drop considerably in proceeding from the ground state geometry to their respective planar optimum geometries; the $1^{2} A_{u}$ state is much less sensitive to the change in geometry. The changes in geometry are consistent with what one would expect upon excitation from $\pi$ bonding to $\pi$ antibonding orbitals, i.e., a lengthening of the $\mathrm{C}-\mathrm{C}$ bonds. We have used the excited state geometries to calculate $0-0$ excitation energies (Table $V$ ). Two basis sets are used and one can see that in the larger basis, the $0-0$ excitation energies differ by at most $0.5 \mathrm{eV}$ from the vertical excitation energies presented in Table II.

Frequency analyses were performed at the planar stationary points for the STO-3 $+\mathrm{G} 3 / 4 \mathrm{CASSCF}$ wave functions. It was found that the $1^{2} B_{g}$ and $1^{2} A_{u}$ states were true minima, but that the $2{ }^{2} B_{g}$ and the $2{ }^{2} A_{u}$ were not, the former having five imaginary frequencies at its stationary point, the latter having one. For the $1^{2} A_{u}$ state, the lowest frequen-

TABLE V. Planar " $0-0$ " excitation energies from $3 / 4$ CASSCF calculations. ${ }^{a}$

\begin{tabular}{ccc}
\hline \hline State & STO-3+G & $6-31 \mathrm{G}$ \\
\hline $1{ }^{2} A_{u}$ & 2.45 & 2.39 \\
$2^{2} A_{u}$ & 4.73 & 4.74 \\
$2^{2} B_{g}$ & 6.30 & 6.27 \\
\hline
\end{tabular}

Excitation energies are in electron-volts relative to the 3/4 CASSCF calculation for the $1{ }^{2} B_{8}$ state in the given basis. The ground state energy for the STO-3 + G calculation is given in Table IV. The geometries used for all calculations in the 6-31G basis are those from the STO-3 + G optimizations, for which the ground state $1^{2} B_{g}$ energy was -154.6148 hartrees. 
TABLE VI. Vertical ionization potentials based on MRSDCI and MRACPF calculations in the DZP + basis."

\begin{tabular}{|c|c|c|c|c|c|c|}
\hline State & Refs. $^{b}$ & \% PTK $^{c}$ & Configurations $^{\mathrm{d}}$ & $\Delta E_{\mathrm{CI}}^{\mathrm{ex}}$ & $\Delta E_{\mathrm{SDCl}}^{\mathrm{ex}}+\mathrm{Q}$ & $\Delta E_{\mathrm{ACPF}}^{\text {ex }}$ \\
\hline$\overline{1^{2} B_{8}}$ & 67 & 93 & 109017 & $8.69(-)$ & $8.79(-)$ & $8.79(-)$ \\
\hline $1^{2} A_{u}$ & 103 & 90 & 173831 & $10.91(2.22)$ & $11.10(2.31)$ & $11.08(2.29)$ \\
\hline $2^{2} A_{u}$ & $97^{e}$ & 83 & 166346 & $13.48(4.79)$ & $13.69(4.90)$ & $13.67(4.88)$ \\
\hline $2^{2} B_{8}$ & $73^{e}$ & 82 & 152864 & $15.17(6.48)$ & $15.19(6.40)$ & $\ldots$ \\
\hline $1^{2} A_{g}$ & 51 & 93 & 110976 & $12.32(3.63)$ & $12.25(3.46)$ & $12.26(3.46)$ \\
\hline $1^{2} B_{w}$ & 123 & 92 & 158189 & $13.56(4.87)$ & $13.56(4.77)$ & $13.57(4.78)$ \\
\hline
\end{tabular}

"Ionization potentials are in electron-volts relative to calculations using a 46 function reference space for the neutral ground state $\left(1^{\prime} A_{g}\right)$ at the experimental ground state geometry, the energies of which are $E_{\mathrm{CI}}^{\text {ex }}$ $=-155.4583$ hartrees; $E_{\mathrm{CI}}^{\mathrm{ex}}+\mathrm{Q}=-155.5257$ hartrees; $E_{\mathrm{ACPF}}^{\mathrm{ex}}=-155.5191$ hartrees. The number of spin-adapted configurations included in the variational portion of the calculation was 81218 . Values in parentheses are excitation energies relative to the $1{ }^{2} B_{g}$ state at this geometry.

${ }^{b}$ The number of spin-adapted configurations in the reference space.

c The percentage of the second-order Rayleigh-Schrödinger perturbation theory energy accounted for by the configurations included in the variational portion of the calculation.

d The number of spin-adapted configurations included in the variational portion of the calculation.

'Two root selection was performed.

cy was on the order of $100 \mathrm{~cm}^{-1}$, half of the lowest frequency for the ground state. Of course, the results for geometries and frequencies are only approximate, due to the small basis and limited correlation treatment used; thus the planarity of this state should be viewed with some caution. For the excited states of the neutral species, it was found that this level of calculation $^{53}$ yielded comparable results to those obtained using larger basis sets and extended correlation treatments, ${ }^{18}$ except that the bond lengths tended to be uniformly long by about $0.02 \AA$. This lends some credence to the present results and it is thus possible that at least one of the lowlying $\pi$ states of $\mathrm{C}_{4} \mathrm{H}_{6}^{+}$is planar, but that deviations from planarity are expected for higher-lying states.

Ionization potentials were calculated using both MRSDCI and MRACPF. The MRSDCI results are given with and without the Davidson size-consistency correction $(+Q)$ discussed above. These calculations were performed at the experimental neutral molecule ground state geometry; thus the results correspond to theoretical estimates of vertical ionizations. In Table VI, it is seen that the three methods used $\mathrm{CI}, \mathrm{CI}+\mathrm{Q}$, and $\mathrm{ACPF}$ yield quite similar estimates of the vertical ionizations, and that the $\mathrm{CI}+\mathrm{Q}$ and $\mathrm{ACPF}$ results are nearly identical. For the ${ }^{2} A_{u} \pi$ states, the I.P.s and excitation energies tend to be slightly higher for $\mathrm{CI}+\mathrm{Q}$ and ACPF, but these differences are small. In most cases, the theoretical ionization potentials are below the experimental results (see the Discussion section). Regarding the spacing between the ion states at the neutral geometry, we underestimate the difference in vertical ionization potentials between the $1^{2} B_{g}$ and $1{ }^{2} A_{u}$ states by about $0.2 \mathrm{eV}$. Compared to electronic absorption measurements, ${ }^{23,25}$ we apparently overestimate the energy difference between the $2^{2} A_{u}$ and $1^{2} B_{g}$ states by at least $0.7 \mathrm{eV}$. In part, this error may arise because we should only compare absorption measurements on the ion to calculations at the ionic ground state geometry if we are concerned with vertical excitation energies. However, results presented below will indicate that even at the ion's equilibrium geometry, this excitation energy is overestimated by at least $0.4 \mathrm{eV}$. This excitation energy was overestimated in the CASSCF results and in all past $a b$ initio studies.

In Table VII, we present MRSDCI and MRACPF cal-

TABLE VII. Vertical excitation energies based on MRSDCI and MRACPF calculations DZP + basis."

\begin{tabular}{lcccccc}
\hline \hline State & Refs. & \%PTK & Configurations & $\Delta E_{\mathrm{CI}}^{\text {ex }}$ & $\Delta E_{\text {SDCI }+Q}^{\text {ex }}$ & $\Delta E_{\text {ACPF }}^{\text {ex }}$ \\
\hline $1{ }^{2} A_{u}$ & $134^{\mathrm{b}}$ & 87 & 202121 & 2.44 & 2.48 & $\ldots$ \\
$2^{2} A_{u}$ & $134^{\mathrm{b}}$ & 86 & 202121 & 4.57 & 4.51 & $\ldots$ \\
$2^{2} B_{g}$ & $114^{\mathrm{b}}$ & 84 & 212161 & 6.42 & 6.38 & 6.36 \\
$1^{2} A_{\mathrm{g}}$ & 153 & 92 & 168960 & 4.42 & 4.39 & 4.39 \\
$11^{2} B_{u}$ & 149 & 91 & 182151 & 5.30 & 5.19 & 5.19 \\
\hline
\end{tabular}

- Excitation energies are in electron-volts relative to calculations using a 112 function reference space for the ionic ground state $\left(1^{2} B_{g}\right)$, the energies of which are $E_{\mathrm{Cl}}^{\mathrm{ex}}=-155.1484$ hartrees; $E_{\mathrm{CI}}^{\mathrm{ex}}+\mathrm{Q}=-155.2084$ hartrees; $E_{\mathrm{ACPF}}^{\mathrm{ex}}=-155.2022$ hartrees. The number of configurations treated variationally for the ground state was 138 470. The labeling is the same as in Table VI.

${ }^{b}$ Two root selection was performed. 
TABLE VIII. Vertical ionization potentials and excitation energies based on MRSDCI and MRACPF calculations, DZP + basis, small reference spaces."

\begin{tabular}{|c|c|c|c|c|c|c|}
\hline State & Refs. & \% PTK & Configurations & $\Delta E_{\mathrm{CI}}^{\mathrm{ex}}$ & $\Delta E_{\mathrm{SDCl}}^{\mathrm{ex}}+\mathrm{Q}$ & $\Delta E_{\mathrm{ACPF}}^{\mathrm{ex}}$ \\
\hline $1^{2} B_{z}$ & 11 & 92 & 69059 & $8.63(-)$ & $8.78(-)$ & $8.78(-)$ \\
\hline $1^{2} A_{k}$ & $29^{b}$ & 82 & 95336 & $10.87(2.23)$ & $11.15(2.36)$ & $11.13(2.35)$ \\
\hline $2^{2} A_{*}$ & $29^{\mathrm{b}}$ & 78 & 95336 & $13.31(4.68)$ & $13.56(4.78)$ & $13.54(4.76)$ \\
\hline $2^{2} B_{z}$ & $14^{b}$ & 86 & 183296 & $15.36(6.73)$ & $15.49(6.71)$ & $\cdots$ \\
\hline $1^{2} A$ & 17 & 91 & 63296 & $12.31(3.67)$ & $12.23(3.45)$ & $12.23(3.45)$ \\
\hline $1^{2} B_{u}$ & 35 & 89 & 78338 & $13.50(4.86)$ & $13.34(4.56)$ & ... \\
\hline
\end{tabular}

- The geometry used is the experimental geometry for the neutral ground state. Ionization potentials are in electron-volts, relative to calculations using seven reference functions on the neutral ground state $\left(1{ }^{1} A_{g}\right)$, the energies of which are $E_{C I}^{\mathrm{ex}}=-155.4538$ hartrees; $E_{\mathrm{CI}+\mathrm{Q}}^{\mathrm{ex}}=-155.5300$ hartrees; $E_{\mathrm{ACPF}}^{\mathrm{ex}}=-155.5228$ hartrees. The number of configurations treated variationally was 58613 . The excitation energies (in parentheses) are relative to the $1^{2} B_{g}$ state at this geometry. The labeling is the same as in Table VI.

b Two root selection was performed.

culations for the same states considered in Table VI, but now at the calculated $1{ }^{2} B_{8}$ geometry of the ion. The $1^{2} A_{u}$ excitation energy increases somewhat (in disagreement with what is observed experimentally), while the $2^{2} A_{u}$ state decreases somewhat in energy, compared to the results at the ground state geometry. The $\sigma$ states undergo the largest change, having significantly higher excitation energies at the ion's ground state geometry.

Table VIII contains results from MRSDCI and MRACPF calculations based on smaller reference spaces than those considered in Tables VI and VII. These calculations are meant to examine how sensitive the above results are to changes in the composition of the reference space, both for total energies and for energy differences. The calculations were all performed at the neutral molecule's ground state geometry. It is seen that the ionization potentials and excitation energies compare reasonably well with the larger reference space results of Table VI, the largest excitation energy difference occurring for the $2{ }^{2} B_{g}$ state. The total energies are somewhat different, but it is doubtless true that the differences are much smaller than the error made due to basis set truncation in this case. Thus, within the limits of the current basis, one could safely perform small reference calculations and achieve accuracy comparable to much larger calculations.
In Table IX, we present MRSDCI and MRACPF results using similar small reference spaces to those employed in Table VIII, but we now use the DZ + basis, i.e., we have removed the polarization functions on all atoms. These calculations were performed at the calculated ground state geometry for the ion and one sees that the energy differences are quite similar to those presented in Table VII. It would appear that removal of the polarization functions does not change the calculated excitation energies significantly.

\section{DISCUSSION}

In this section, we discuss the results obtained in light of available experimental data and past theoretical work. We also compare the various theoretical methods used here.

\section{A. Experiment}

The threshold for ionization from $\mathrm{C}_{4} \mathrm{H}_{6}$ to yield the $1^{2} B_{g}$ state of the ion has been estimated to be $9.07 \mathrm{eV} .{ }^{54,55}$ The present results uniformly underestimate this; the $\mathrm{CI}+\mathrm{Q}$ and $\mathrm{ACPF}$ values are slightly better than the CI values, but their errors are still of comparable magnitude. One notes that the IP in Table VI is a vertical ionization potential and that allowing geometrical relaxation in the ion will only exacerbate the error. The CASSCF result also un-

TABLE IX. Vertical excitation energies based on MRSDCI and MRACPF calculations, DZ + basis, small reference spaces."

\begin{tabular}{ccccccc}
\hline \hline State & Refs. & \%PTK & Configurations & $\Delta E_{\mathrm{CI}}^{\mathrm{ex}}$ & $\Delta E_{\mathrm{SDCl}+\mathrm{Q}}^{\mathrm{ex}}$ & $\Delta E_{\mathrm{ACPF}}^{\mathrm{ex}}$ \\
\hline $1^{2} A_{4}$ & $10^{\mathrm{b}}$ & 97 & 86627 & 2.58 & 2.52 & 2.52 \\
$2^{2} A_{*}$ & $10^{\mathrm{b}}$ & 97 & 86627 & 4.59 & 4.45 & 4.45 \\
$2^{2} B_{\mathrm{z}}$ & $14^{\mathrm{b}}$ & 94 & 109775 & 6.50 & 6.44 & 6.43 \\
\hline \hline
\end{tabular}

\footnotetext{
- Excitation energies are in electron-volts, relative to calculations using eight reference functions for the ionic ground state $\left(1^{2} B_{q}\right)$, the energies of which are $E_{\mathrm{Cl}}^{\mathrm{ex}}=-154.9113$ hartrees; $E_{\mathrm{CI}}^{\mathrm{ex}}+\mathrm{Q}=-154.9509$ hartrees; $E_{A C P F}^{\text {.X }}=-154.9468$ hartrees. The number of configurations treated variationally was 35073 . The labeling is the same as in Table VI.

'Two root selection was performed.
} 
TABLE X. Previous estimates of ion-state energy differences. ${ }^{2}$

\begin{tabular}{|c|c|c|c|c|}
\hline \multicolumn{3}{|l|}{ State } & \multicolumn{2}{|l|}{$\Delta E$} \\
\hline \multicolumn{5}{|c|}{ Experimental results } \\
\hline $1^{2} A_{u}$ & $2.46{ }^{b}$ & $2.3,^{\mathrm{c}}$ & $2.3,^{\mathrm{d}}$ & $2.45^{\mathrm{e}}$ \\
\hline $2^{2} A_{u}$ & & $4.0^{c}$ & & $4.2^{\mathrm{e}}$ \\
\hline $2^{2} B_{g}$ & & & $\cdots$ & \\
\hline $1^{2} A_{g}$ & $3.26,^{b}$ & & & $3.2^{\mathrm{e}}$ \\
\hline $1^{2} B_{u}$ & $4.6,{ }^{b}$ & & & $4.7^{e}$ \\
\hline \multicolumn{5}{|c|}{ Semiempirical theoretical results } \\
\hline $1^{2} A_{u}$ & 2.22, & $1.8{ }^{\mathrm{f}}$ & $2.7,8$ & $2.8^{\mathrm{h}}$ \\
\hline $2^{2} A_{u}$ & $3.47,^{\mathrm{d}}$ & $3.0,{ }^{f}$ & $4.6,8$ & $3.8^{\mathrm{h}}$ \\
\hline $2^{2} B_{g}$ & & & $\cdots$ & \\
\hline $1^{2} A_{g}$ & $2.3,^{d}$ & $2.3,^{f}$ & $3.2,8$ & $4.0^{\mathrm{h}}$ \\
\hline $1^{2} B_{u}$ & & $3.7^{\mathrm{f}}$ & & \\
\hline \multicolumn{5}{|c|}{ Ab initio results } \\
\hline $1^{2} A_{u}$ & & 2.7, & & $2.8^{j}$ \\
\hline $2^{2} A_{u}$ & & 5.8, & & $5.7^{j}$ \\
\hline $2^{2} B_{g}$ & & & $\cdots$ & \\
\hline $1^{2} A_{8}$ & & 3.7, & & $3.5^{\mathrm{j}}$ \\
\hline $1^{2} B_{4}$ & & $5.1,{ }^{i}$ & & $5.1^{j}$ \\
\hline
\end{tabular}

${ }^{2}$ For photoelectron spectrum data, the IP differences were converted to ion state energy differences by subtracting the lowest IP from all higher values.

${ }^{b}$ Reference 21 , UPS.

${ }^{c}$ Reference 23, PDS.

${ }^{\mathrm{d}}$ Reference 25, UV/visible in Ar.

'Reference 24

${ }^{\mathrm{f}}$ Reference 30(a), modified neglect of diatomic overlap (MNDO) estimated from graphs.

${ }^{8}$ Reference 30 (b), local neglect of differential overlap.

' Reference 32, CNDO with limited CI.

'Reference 33, Green's function estimated from graphs.

${ }^{j}$ Reference 34, CI.

derestimates the ionization potential. This error in all cases should be taken as an indication of the accuracy one might expect from calculations at this level. Ionization potentials and electron affinities can be somewhat more difficult to calculate than excitation energies due to the differing number of electrons and thus different correlation energies in the two states, and additional errors surely arise from the limited one-electron basis. Thus, when one obtains highly accurate results for a given excitation energy, it is well to realize that high accuracy may result from a fortuitous cancellation of errors.

A summary of past experimental, semiempirical, and $a b$ initio results for energy differences between ion states is presented in Table X. For ultraviolet photoelectron (UPS) data, we have converted the IP differences to excitation energies by subtracting the lowest IP from all others. While these are not identical to vertical excitation energies for the ion, it allows easier comparison among the various methods.

Eland $e t$ al. $^{21}$ and Brundle and Robin ${ }^{22}$ have published estimates of higher-lying ionizations and assignments of these features. They assign vertical IPs of approximately $11.5 \mathrm{eV}\left(1^{2} A_{u}\right), 12.3 \mathrm{eV}\left(1^{2} A_{g}\right)$, and $13.5-13.7 \mathrm{eV}\left(1^{2} B_{u}\right)$. In contrast to the first ionization, the remaining bands have less discernible structure. Our accuracy in these cases is similar to that obtained for the first ionization. The $2^{2} A_{u}$ state is supposed to be of mixed character, but is unassigned. The
$2^{2} B_{g}$ is also unassigned, and due to its non-Koopmans' character, one expects it to have a weak intensity in a photoelectron spectrum.

Studies of excitation spectra for the butadiene radical cation have been published either as absorption spectra in matrices $^{25}$ or as photodissociation spectra in the gas phase. ${ }^{23}$ The prominent features have been assigned to the $1^{2} A_{u}$ and $2^{2} A_{u}$ states, at $2.2-2.4$ and $4.0-4.2 \mathrm{eV}$, respective1y. ${ }^{25}$ The value for the $1^{2} A_{u}$ state is in reasonable agreement with the results presented here (approximately $2.5 \mathrm{eV}$ ). The vertical transition to the $2^{2} A_{u}$ state is calculated to be about $0.7-0.9 \mathrm{eV}$ higher than observed experimentally at the CASSCF level and from 0.3 to $0.6 \mathrm{eV}$ higher at the MRSDCI or MRSDCI + $Q$ levels. It is possible that nonvertical contributions to this transition give rise to a significant portion of the intensity as has been seen in ethylene $e^{56}$ and has been postulated for butadiene. ${ }^{17(a)}$ Indeed, the geometry optimization suggests that the planar configuration is not a minimum, but a transition state. However, unlike ethylene or butadiene, this state is valencelike (not Rydberg-like at the ground state geometry) and thus one should still see significant intensity for the vertical excitation. While our excitation energies are below those of previous $a b$ initio studies, they are still above the experimental value; the reason for this is not obvious.

Interestingly, $a b$ initio theory currently overestimates the excitation energy to the $2{ }^{1} A_{g}$ state for the neutral polyenes. Recent work on cis-hexatriene ${ }^{7}$ and octatetraene ${ }^{11}$ has located the $2^{1} A_{g}$ state at least $1 \mathrm{eV}$ below that of large scale $a b$ initio results. ${ }^{17(b), 20}$ (In the case of hexatriene, we compare here with theoretical results on the all trans species. Based on results for butadiene, ${ }^{17(a)}$ large differences are not expected to arise.) This state is also a multiconfigurational valencelike state, and in comparison with semiempirical results, $a b$ initio studies find it uniformly higher in energy. The reason for this discrepancy is not clear yet. It is true that for such multiconfigurational states we have not been able to recover as large a portion of the correlation energy variationally as in the predominantly single configurational states. If $\sigma \sigma^{\prime}$ or $\sigma \pi$ correlation is particularly important for these states, we would tend to overestimate their excitation energies. However, for the neutral species, unselected calculations have been performed for the $2^{1} A_{g}$ state ${ }^{18}$ (using the interacting space approximation) and they yield results quite similar to the largest selected results, ${ }^{17(a)}$ suggesting that the selection procedure may be reasonably accurate.

Another point of disagreement with experiment occurs in comparing the $1^{2} B_{g} \rightarrow 1^{2} A_{u}$ energy differences at the neutral and cation geometries. The intensity maxima in the experimental spectra suggest that this difference decreases in going to the ion geometry. Our extended correlation results suggest it should increase somewhat and the CASSCF results also predict an (albeit smaller) increase. Whether the experimental differences arise from nonvertical contributions at the intensity maxima is not known at present. The errors may also arise from inaccuracies in the molecular geometries. Finally, the errors may merely arise from the fact that the "inherent error" in the present calculations, based on the IP errors, is on the order of $0.4 \mathrm{eV}$ and the difference 
seen here falls within this realm.

No intensity is observed for transitions to the $\sigma$-hole states in the absorption experiments; thus it is not possible at present to determine if our higher estimates of the $1^{2} B_{8} \rightarrow 1{ }^{2} A_{8}, 1^{2} B_{u}$ transitions at the ionic ground state geometry are reasonable or not. However, we predict that these states occur at significantly higher energies relative to the $1^{2} B_{g}$ state at the ionic ground state than they do at the neutral's geometry.

Regarding $0-0$ transition energies, the CASSCF results suggest that the $\pi$ states do not drop significantly in energy in moving to their planar stationary points. This is in agreement with the electronic absorption experiments ${ }^{23,25}$ for the $1^{2} A_{u}$ state, where the vertical and $0-0$ transitions are separated little in energy. In the case of the $2^{2} A_{u}$ state, the separation between the band maximum and its origin is somewhat greater, but it is possible that this low-energy intensity arises from nonplanar configurations which were not considered here. This prediction of little rearrangement energy upon relaxation in the plane for such multiconfigurational excited states is in contrast to semiempirical and ab initio results for the neutral molecule, especially for the multiconfigurational $2^{2} A_{g}$ state, which all past theory has suggested decreases by up to $1 \mathrm{eV}$ upon relaxation to a planar stationary point.

In summary then, the present results are in relatively good agreement with experiment for all states except for $2^{2} A_{u}$, for which our results are high by from 0.4 to $0.6 \mathrm{eV}$.

\section{B. Past theory}

Since past semiempirical theory ${ }^{25,30-32}$ has been used in assigning experimental spectra, it will not be a surprise that similar comparisons can be made here as were made with experiment. Most past work has either dealt with transitions at the neutral geometry, appropriate to photoionization, or has used approximate geometries and the distinction between ionization energy differences and absorption spectra estimates is blurred somewhat. In general, one finds that the previous semiempirical results ${ }^{25}$ tend to yield lower excitation energies than the present or past $a b$ initio results. One of the semiempirical calculations is much closer to the experimental intensity maximum for the $1^{2} A_{u}$ state, the others are either too low or too high by amounts comparable to the errors obtained here.

Comparing our results with past $a b$ initio studies, ${ }^{33,34}$ we obtain lower excitation energies for the positive ion, with the major differences occurring for the excited $\pi$ states. Comparison of the excitation energies is the more clearcut comparison to make, since one can obtain arbitrarily low or high ionization estimates just by a poor treatment of the differential correlation energy between the neutral and the ion. A variety of factors may have led to our results being closer to experiment, among which are the separately optimized orbitals for each state, more extensive correlation treatment (MRSDCI vs SDCI ${ }^{34}$ or single reference Green's function procedure ${ }^{33}$ ), and larger basis set (polarization functions and Rydberg functions).

\section{Comparison among the present methods}

It is seen that in all cases, the MRSDCI, MRSDCI + Q, or MRACPF methods yield vertical I.P.s or excitation energies, which are in closer agreement with experiment than the CASSCF results. The CASSCF excitation energies are generally somewhat higher, with the largest differences occurring for the $1^{2} B_{u}$ and $2^{2} A_{u}$ states. In part, this may occur because the $\sigma$ electrons are not correlated in these calculations and this will tend to yield overly large stretching force constants for the ground and excited states. Since the ground state equilibrium geometry is used for the calculations, the largest errors will occur for excited states, yielding excitation energies that are too high. In addition, if differential $\sigma \sigma$ and/or $\sigma \pi$ correlation is important in determining the excitation energies, one expects errors at the CASSCF level used here. These effects seem to be of the order of $0.5 \mathrm{eV}$ for most states, except the $1^{2} B_{u}$ state, where the CASSCF excitation energy is nearly an electron-volt higher than the results of Table VII. One could argue, however, that the increased accuracy is more of a quantitative nature than qualitative. That is, other than for the relative placement of the $1{ }^{2} B_{u}$ and $2^{2} A_{u}$ states at the neutral geometry, no new assignments would arise from the higher level. The MRSDCI calculations took on average 20 to 30 times longer than the CASSCF results and it is useful to see that one can obtain moderately accurate results quickly.

Comparing the MRSDCI results with the MRSDCI + $Q$ or MRACPF, one sees that there are small differences between the size-inconsistent and size-consistency corrected results. The IP to form the ground state ion is increased by $0.1 \mathrm{eV}$ by inclusion of some form of size-consistency correction and there tend to be differences of this order for other excitation energies or IPs. These differences are relatively minor, however, and suggest that though the MRSDCI results have a fairly large size inconsistency (compare the $1{ }^{2} B_{g}$ energies with and without some correction for size inconsistency), the relative energies are only marginally affected.

More striking perhaps are the nearly identical excitation energies and IPs obtained by MRSDCI $+Q$ and MRACPF. The + $\mathrm{Q}$ correction for MRSDCI $+\mathrm{Q}$ is applied after performance of a conventional $\mathrm{CI}$ and is based on the (sizeinconsistent) $\mathrm{CI}$ coefficients and energy. The MRACPF energy is obtained via similar reasoning to that used in obtaining the Davidson correction, but the modifications are made to the energy functional itself, before the calculation. One might reason that as the size of the system increased, the Davidson correction should deteriorate due to the size inconsistency of the "input" to the correction, but no such deterioration is seen here, in comparison with ACPF. Of course, the near agreement of the excitation energies from the uncorrected MRSDCI results with either of the "sizeconsistent" results may also be masking this effect, but this does give one hope that reasonably accurate size-consistency corrected energy differences can be obtained from the $\mathrm{Da}$ vidson corrected results with little cost.

This last result is of interest for several reasons. One is that ACPF or quasidegenerate variational perturbation the- 
ory ${ }^{46}$ calculations (a method related to ACPF) tend to be susceptible to "intruder-state" problems. ${ }^{46,57,58}$ These problems arise when a configuration outside the reference space yields an artificially large coefficient in the expansion, but upon inclusion in the reference space, its coefficient drops to a "normal" size again. Single excitations most often occur as intruder states, because the zeroth-order energy of such lowlying configurations (or that of a low-lying configuration interacting with the other single and double excitations) approaches that of the zeroth-order wave function. Configuration interaction is much less susceptible to this problem and as a result tends to be more robust. Another positive aspect of the accuracy of the Davidson correction for systems of this type is that one can assess easily whether size inconsistency will be significant or not for excitation energies by performing $\mathrm{Cl}$ calculations. One can then decide whether to perform more extensive size-consistent calculations. In any event, these results suggest that the simple Davidson correction to MRSDCI is of comparable quality to what would appear to be a more substantive approach, at least for systems of the size studied here.

Finally, the above results indicate that smaller reference space calculations were capable of reproducing the larger reference results in most cases. Comparing Table VIII to Table VI, one sees that while total energies differed to some degree, the excitation energies obtained with the smaller reference spaces are in reasonably good agreement in most cases. In a similar vein, no new qualitative results are obtained by neglect of polarization functions in the MRSDCI and MRACPF calculations. Given the level of variational recovery of correlation and the level of the basis set used, one would have to say that the results are not significantly different.

One could expand significantly the variational correlation recovery for the MRSDCI and MRACPF calculations performed here. At present, we have not been able to go beyond the levels reported due to computational limitations, but it is well to realize that the extrapolation procedure used here varies in accuracy as the fraction of the second-order energy recovered changes. ${ }^{59}$ Thus inaccuracies in the extrapolation could also contribute to differences relative to experiment.

\section{CONCLUSIONS}

Results are presented from $a b$ initio calculations for the ground and several excited states of the butadiene radical cation. Optimized planar geometries are presented at the CASSCF level in different basis sets, and in the STO-3 + G basis estimated planar geometries were presented for $\pi$ excited states. In general, it is seen that bond alternation is significantly less in the cation than in the neutral. Extended MRSDCI and MRACPF calculations were performed on various $\sigma$ and $\pi$ excited states, and while these results were quantitatively closer to the experimentally assigned states, no qualitative differences were obtained relative to the CASSCF results. It is found that all $a b$ initio methods appear to overestimate the excitation energy for the $2^{2} A_{u}$ excited state, while at least one semiempirical method is in much closer agreement with experiment. An assignment is also made for the previously unobserved $2{ }^{2} B_{g}$ state.

The relative accuracy of the various methods is also compared and it is found that the MRACPF and MRSDCI + $Q$ results are in the best agreement with experiment. These two methods yield essentially indistinguishable excitation energies, even though the former includes corrections for size inconsistency at the outset of the calculation, whereas the latter only includes such effects after performance of a CI. Uncorrected CI is nearly as accurate; we find that size inconsistency has a smaller effect on the excitation energies for the cation than the neutral species. However, it should be noted that size inconsistency still has a large effect on the total energy estimates. Finally, while the CASSCF energies tend to be somewhat higher than the more extensive correlation methods, especially for the $\sigma$ states, it is nevertheless the case that it yields correct state orderings within the $\sigma$ and $\pi$ manifolds. We believe that CASSCF results should be adequate for examination of the relative excitation energies of bond rotation isomers of polyene radical cations and are currently studying the hexatriene cation using CASSCF wave functions. ${ }^{60}$

\section{ACKNOWLEDGMENTS}

Acknowledgment is made to the Donors of the Petroleum Research Fund, administered by the American Chemical Society, for partial support of this research. This research was also partially supported by a grant from the National Science Foundation, Grant No. CHE-9011770. The calculations were performed on the Harvey Mudd Computational Chemistry Facility, the establishment of which was made possible by a grant from the National Science Foundation, Grant No. USE-8950661.

${ }^{1}$ R. R. Birge, Annu. Rev. Biophys. Bioeng. 10, 315 (1981).

${ }^{2}$ J. L. Bredas and G. B. Street, Acc. Chem. Res. 18, 309 (1985).

${ }^{3}$ B. S. Hudson, B. E. Kohler, and K. Schulten, Excited States 6, 1 (1982)

${ }^{4}$ B. S. Hudson and B. E. Kohler, Chem. Phys. Lett. 14, 299 (1972); J. Chem. Phys. 59, 4984 (1973).

${ }^{5}$ R. M. Gavin, Jr., C. Weisman, J. K. McVey, and S. A. Rice, J. Chem. Phys. 68, 522 (1978).

${ }^{6}$ B. S. Hudson and B. E. Kohler, Annu. Rev. Phys. Chem. 25, 437 (1974).

${ }^{7}$ W. J. Buma, B. E. Kohler, and K. Song, J. Chem. Phys. 92, 4622 (1990); 94, 6367 (1991).

${ }^{8}$ R. R. Chadwick, D. P. Gerrity, and B. S. Hudson, Chem. Phys. Lett. 73, 5508 (1980).

${ }^{9}$ O. A. Mosher, W. M. Flicker, and A. Kuppermann, J. Chem. Phys. 59, 6502 (1973).

${ }^{10} \mathrm{~J}$. P. Doering and R. McDiarmid, J. Chem. Phys. 75, 2477 (1981).

${ }^{11}$ M. F. Granville, G. R. Holtom, and B. E. Kohler, J. Chem. Phys. 72, 4671 (1980).

${ }^{12}$ (a) K. Schulten, I. Ohmine, and M. Karplus, J. Chem. Phys. 64, 4422 (1976); (b) P. Tavan and K. Schulten, ibid. 70, 5407 (1979); (c) A. C. Lasaga, R. J. Aerni, and M. Karplus, ibid. 73, 5230 (1980).

${ }^{13}$ R. J. Buenker and J. L. Whitten, J. Chem. Phys. 49, 5381 (1969).

${ }^{14}$ R. P. Hosteny, T. H. Dunning, Jr., R. R. Gilman, A. Pipano, and I. Shavitt, J. Chem. Phys. 62, 4764 (1975).

${ }^{15}$ (a) S. Shih, R. J. Buenker, and S. D. Peyerimhoff, Chem. Phys. Lett. 16, 244 (1972); (b) R. J. Buenker, S. Shih, and S. D. Peyerimhoff, ibid. 44, 385 (1976).

${ }^{16}$ (a) M. A. C. Nascimento and W. A. Goddard III, Chem. Phys. 36, 147 (1979); (b) 53, 251 (1980); (c) Chem. Phys. Lett. 60; 197 (1979).

${ }^{17}$ (a) R. J. Cave and E. R. Davidson, J. Phys. Chem. 91, 4481 (1987); (b) 92, 614 (1988).

${ }^{18}$ P. G. Szalay, A. Karpfen, and H. Lischka, Chem. Phys. 130, 219 (1989). 
${ }^{19}$ R. J. Cave and E. R. Davidson, Chem. Phys. Lett. 148, 190 (1988).

${ }^{20}$ R. J. Cave and E. R. Davidson, J. Phys. Chem. 92, 2173 (1988).

${ }^{21}$ J. H. Eland, Int. J. Mass Spectrom. Ion Phys. 2, 471 (1969).

${ }^{22}$ C. R. Brundle and M. B. Robin, J. Am. Chem. Soc. 92, 5550 (1970).

${ }^{23}$ R. C. Dunbar, Chem. Phys. Lett. 32, 508 (1975).

${ }^{24}$ T. Koenig, C. E. Klopfenstein, S. Southworth, J. A. Hoobler, R. A. Wielesek, T. Balle, W. Snell, and D. Imre, J. Am. Chem. Soc. 105, 2256 (1983).

${ }^{25}$ T. Bally, S. Nitsche, K. Roth, and E. Haselbach, J. Am. Chem. Soc. 106, 3927 (1984).

${ }^{20} \mathrm{~F}$. Gerson and X.-Z. Qin, Helv. Chim. Acta 71, 1065 (1988).

${ }^{27}$ J. N. Aebischer, T. Bally, K. Roth, E. Haselbach, F. Gerson, and X.-Z. Qin, J. Am. Chem. Soc. 111, 7909 (1989).

${ }^{28}$ M. Beez, G. Bieri, H. Bock, and E. Heilbronner, Helv. Chim. Acta 56, 1028 (1973).

${ }^{29} \mathrm{I}$. Duncan and L. Andrews, Tetrahedron 41, 145 (1985).

${ }^{30}$ (a) R. Schulz, A. Schweig, and W. Zittlau, J. Am. Chem. Soc. 105, 2980 (1983); (b) Chem. Phys. Lett. 106, 467 (1984).

"R. W. Bigelow, Int. J. Quantum Chem. 29, 35 (1986).

${ }^{32}$ R. Zahradnik, P. Carsky, and Z. Slania, Coll. Czech. Chem. Commun. 38, 1886 (1973).

${ }^{33}$ L. S. Cederbaum, W. Domcke, J. Schirmer, W. von Niessen, G. H. F. Diercksen, and W. P. Kramer, J. Chem. Phys. 69, 1591 (1978).

${ }^{34}$ K. Kimura, S. Katsumata, Y. Achiba, T. Yamazaki, and S. Iwata, Handbook of HeI Photoelectron Spectra of Fundamental Organic Molecules (Halstead, New York, 1981), pp. 62-63.

${ }^{35}$ R. Gdanitz and R. Ahlrichs, Chem. Phys. Lett. 143, 413 (1988).

${ }^{36}$ E. R. Davidson and L. E. McMurchie, Excited States, 5, 1 (1982).

${ }^{37}$ R. J. Bartlett, Annu. Rev. Phys. Chem. 73, 5711 (1980).

${ }^{38}$ W. Haugen and M. Traetteberg, Acta Chem. Scand. 20, 1726 (1966).

${ }^{39}$ (a) W. J. Hehre, R. F. Stewart, and J. A. Pople, J. Chem. Phys. 51, 2657 (1969); (b) W. J. Hehre, R. Ditchfield, R. F. Stewart, and J. A. Pople, ibid. 52, 2769 (1970).

${ }^{40}$ (a) R. Ditchfield, W. J. Hehre, and J. A. Pople, J. Chem. Phys. 54, 724 (1971); (b) 56, 2257 (1972); (c) P. C. Hariharan and J. A. Pople, Theor. Chim. Acta 28, 213 (1973).

" (a) T. H. Dunning, Jr., J. Chem. Phys. 53, 2823 (1970); (b) S. Huzinaga, ibid. 42, 1293 (1965); (c) T. H. Dunning, Jr. and P. J. Hay, in Methods of Electronic Structure Theory, edited by H. F. Schaefer III (Plenum, New
York, 1977), p. 1.

${ }^{42}$ D. C. Rawlings, E. R. Davidson, and M. Gouterman, Int. J. Quantum Chem. 26, 251 (1984).

${ }^{43}$ S. R. Langhoff and E. R. Davidson, Int. J. Quantum Chem. 8, 61 (1974).

${ }^{44}$ E. R. Davidson and D. W. Silver, Chem. Phys. Lett. 53, 403 (1977).

${ }^{45}$ M. A. Anderson and R. J. Cave, Chem. Phys. 154, 1 (1991).

${ }^{46}$ R. J. Cave and E. R. Davidson, J. Chem. Phys. 89, 6798 (1988).

${ }^{47}$ D. Feller and E. R. Davidson, J. Chem. Phys. 74, 3977 (1981).

${ }^{48}$ E. R. Davidson, Rev. Mod. Phys. 44, 451 (1972).

${ }^{49}$ C. F. Bender and H. F. Schaefer III, J. Chem. Phys. 55, 4798 (1971).

${ }^{50}$ GAMESS is a general purpose electronic structure program. The original program was assembled by M. Dupuis, D. Spangler, and J. J. Wendoloski at the National Resource for Computations in Chemistry, Software Catalog, University of California, Berkeley, CA, 1980, program QGO1. The current version is described in the Quantum Chemistry Program Exchange Newsletter: M. W. Schmidt, K. K. Baldridge, J. A. Boatz, J. H. Jensen, S. Koseki, M. S. Gordon, K. A. Nguyen, T. L. Windus, and S. T. Elbert, QCPE Bull. 10, 52 (1990).

${ }^{51}$ The MELDF series of electronic structure codes was developed by $L$. E. McMurchie, S. T. Elbert, S. R. Langhoff, E. R. Davidson, and D. Feller, and was modified extensively by D. C. Rawlings.

52 (a) M. Aoyagi, Y. Osamura, and S. Iwata, J. Chem. Phys. 83, 1140 (1985); (b) M. Aoyagi and Y. Osamura, J. Am. Chem. Soc. 111, 470 (1989).

${ }^{53}$ P. G. Szalay, H. Lischka, and A. Karpfen, J. Phys. Chem. 93, 6629 (1989).

${ }^{54}$ T. Reddish, B. Wallbank, and J. Comer, Chem. Phys. 108, 159 (1986).

${ }^{55}$ R. McDiarmid, J. Chem. Phys. 64, 514 (1976).

${ }^{56}$ C. Petrongolo, R. J. Buenker, and S. D. Peyerimhoff, J. Chem. Phys. 76, 3655 (1982).

${ }^{57}$ S. Evangelisti, J. P. Daudey, and J.-P. Malrieu, Phys. Rev. A 35, 4930 (1987).

${ }^{58}$ C. W. Bauschlicher, Jr., S. R. Langhoff, and A. Korminicki, Theor. Chim. Acta 77, 263 (1990).

${ }^{59}$ R. J. Cave, S. Xantheas, and D. Feller, Theor. Chim. Acta. (to be published).

${ }^{60}$ R. J. Cave and J. L. Johnson, J. Phys. Chem. (submitted for publication). 